\title{
Molecular modeling of hydrogen bonding fluids: Vapor-liquid coexistence and interfacial properties
}

\author{
Martin Horsch ${ }^{1}$, Martina Heitzig ${ }^{2}$, Thorsten Merker $^{3}$, Thorsten Schnabel ${ }^{2}$, Yow-Lin \\ Huang ${ }^{1}$, Hans Hasse ${ }^{3}$, and Jadran Vrabec ${ }^{1}$ \\ 1 Lehrstuhl für Thermodynamik und Energietechnik (ThEt), Universität Paderborn, \\ Warburger Str. 100, 33098 Paderborn, Germany ${ }^{\dagger}$ \\ 2 Institut für Technische Thermodynamik und Thermische Verfahrenstechnik (ITT), \\ Universität Stuttgart, Pfaffenwaldring 9, 70569 Stuttgart, Germany \\ 3 Lehrstuhl für Thermodynamik (LTD), Technische Universität Kaiserslautern, \\ Erwin-Schrödinger-Str. 44, 67663 Kaiserslautern, Germany
}

\section{Introduction}

A major challenge for molecular modeling consists in optimizing the unlike interaction potentials. A broad study on fluid mixtures [1] recently showed that among the variety of combination rules that were proposed in the past, none is clearly superior. In many cases, all are suboptimal when accurate predictions of properties like the mixture vapor pressure are needed. The well known Lorentz-Berthelot rule performs quite well and can be used as a starting point. If more accurate results are required, it is often advisable to adjust the dispersive interaction energy parameter which leads to very favorable results $[1,2,3,4,5]$.

A similar approach should be followed for effective pair potentials acting between fluid particles and the atoms of a solid wall. They can only be reliable if fluid-wall contact effects are taken into account, e.g., by adjusting unlike parameters to contact angle measurements [6]. Teletzke et al. [7] used continuum methods to examine the dependence of wetting and dewetting transitions on characteristic size and energy parameters of the fluid-wall dispersive interaction. MD simulation can be applied for the same purpose, leading to a consistent molecular approach.

On the molecular level, the precise position of the vapor-liquid phase boundary is defined by a cluster criterion. Many different criteria are known and it is not immediately obvious which of them leads to the most accurate results [8]. In nanoscopic systems, minute absolute differences can lead to comparably large relative deviations. Therefore, the viability of several criteria is compared in the present study with the purpose of excluding errors due to an inaccurate detection of the interface.

\footnotetext{
${ }^{\dagger}$ Author to whom correspondence should be addressed: Prof. Dr.-Ing. habil. J. Vrabec. E-mail: jadran.vrabec@upb.de.
} 
If the cohesion of the liquid phase is partly due to hydrogen bonds, successful molecular models for pure fluids can often be developed on the basis of an ab initio study of the charge distribution as well as the equilibrium position of the nuclei. This sterically realistic approach, combined with adjusting the Lennard-Jones (LJ) potential parameters to vapor-liquid equilibrium (VLE) data, leads to empirical models that correctly reproduce and predict thermophysical fluid properties over a wide range of conditions [9]. The present work applies this approach to mixtures containing hydrogen bonding components. Often potential parameters determined for one fluid carry over to a derivative with different substituents, opening the possibility of creating generic molecular models. Such a model is presented for benzyl alcohol.

The following publications in peer-reviewed international journals contribute to the present project:

- Schnabel, T., Vrabec, J. \& Hasse, H. Molecular simulation study of hydrogen bonding mixtures and new molecular models for mono- and dimethylamine. Fluid Phase Equilib. 263: 144-159 (2008).

- Eckl, B., Vrabec, J. \& Hasse, H. An optimized molecular model for ammonia. Mol. Phys. 106: 1039-1046 (2008).

- Eckl, B., Vrabec, J. \& Hasse, H. Set of molecular models based on quantum mechanical ab initio calculations and thermodynamic data. J. Phys. Chem. B 112: 12710-12721 (2008).

- Vrabec, J., Huang, Y.-L. \& Hasse, H. Molecular models for 267 binary mixtures validated by vapor-liquid equilibria: a systematic approach. Fluid Phase Equilib. 279: 120-135 (2009).

- Huang, Y.-L., Vrabec, J. \& Hasse, H. Prediction of ternary vapor-liquid equilibria for 33 systems by molecular simulation. Fluid Phase Equilib. 287: 62-69 (2009).

- Horsch, M., Heitzig, M., Dan, C., Harting, J., Hasse, H., Fischer, J. \& Vrabec, J. Contact angle dependence on the fluid wall dispersive energy. In preparation.

It would exceed the scope of the present report to give a full exposition of these articles. Instead, a few points are emphasized and arranged as follows: Firstly, mixture properties are explored for binary systems containing hydrogen bonding components. Secondly, vapor-liquid interface cluster criteria and contact angles are discussed and remarks on computational details are given. Finally, a sterically accurate generic model for benzyl alcohol is introduced and evaluated.

\section{Fluid mixtures with hydrogen bonding components}

Vapor-liquid equilibria of 31 binary mixtures consisting of one hydrogen bonding and one non-hydrogen bonding component were studied. All models are of the rigid united-atom multi-center LJ type with superimposed electrostatic sites in which hydrogen bonding is described by partial charges. The hydrogen bonding components of the studied binary mixtures are: monomethylamine (MMA) and dimethylamine (DMA), methanol, ethanol and formic acid. The non-hydrogen bonding components are: neon, argon, krypton, xenon, methane, oxygen, nitrogen, carbon dioxide, ethyne, 
ethene, ethane, propylene, carbon monoxide, diflourodichloromethane (R12), tetraflouromethane (R14), diflourochloromethane (R22), diflouromethane (R32), 1,1,1,2tetraflouroethane (R134a) and 1,1-diflouroethane (R152a).

To obtain a quantitative description of the mixture vapor-liquid equilibria, one state independent binary interaction parameter was adjusted to a single experimental data point of either the vapor pressure or the Henry's law constant. Throughout, excellent predictions were found at other state points, i.e., at other compositions or temperatures as well as for the Henry's law constant, if it was adjusted to the vapor pressure, or vice versa. Figures 1 and 2 show methane + methanol as a typical example.

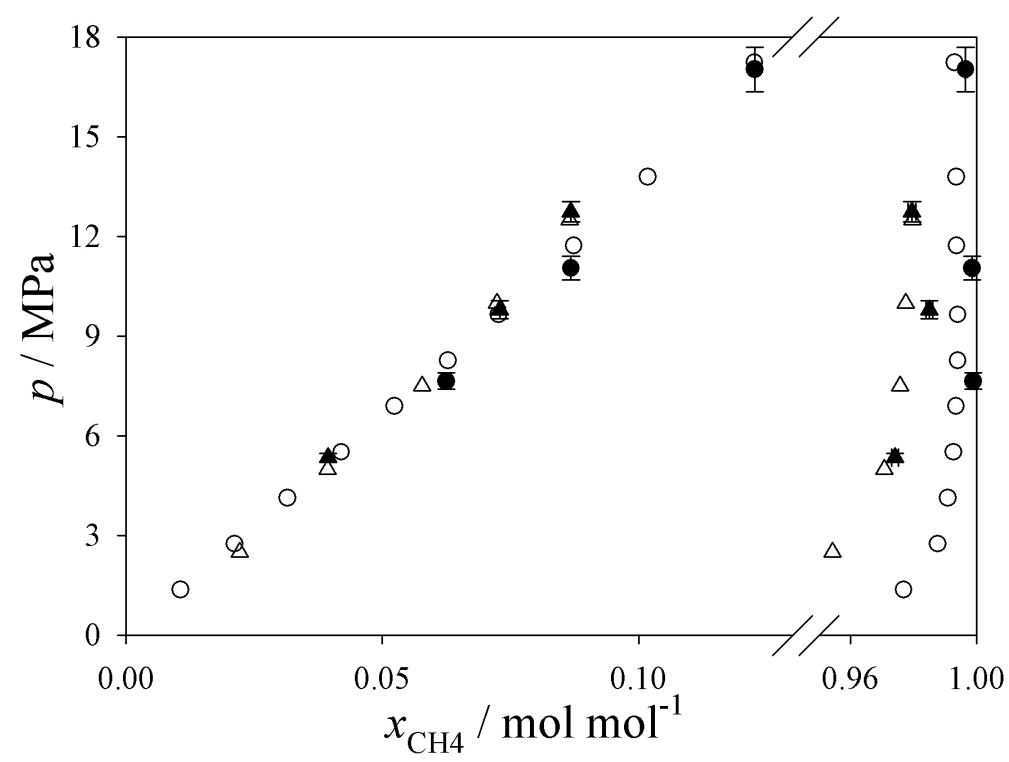

Fig. 1. Simulation data and vapor-liquid equilibria of methanol + methane: simulation data $(\bullet)$ and experimental data (०) at $310 \mathrm{~K}$ [10]; simulation data ( $\mathbf{\Delta})$ and experimental data $(\Delta)$ at $338.15 \mathrm{~K}$ [11].

Furthermore, a set of 78 pure substances from prior work [22, 23] was taken to systematically describe all 267 binary mixtures of these components for which relevant experimental VLE data are available. Again, per binary system, the single state independent binary interaction parameter in the energy term was adjusted to only one experimental value of the vapor pressure. The unlike energy parameter was thereby altered usually by less than $5 \%$ from the Berthelot rule. The mixture models were validated regarding the vapor pressure at other state points as well as the dew point composition, which is a fully predictive property in this context. In almost all, i.e., $97 \%$ of the cases, the molecular models give excellent predictions of the mixture properties. Compared to other works in the literature, this is by far the 


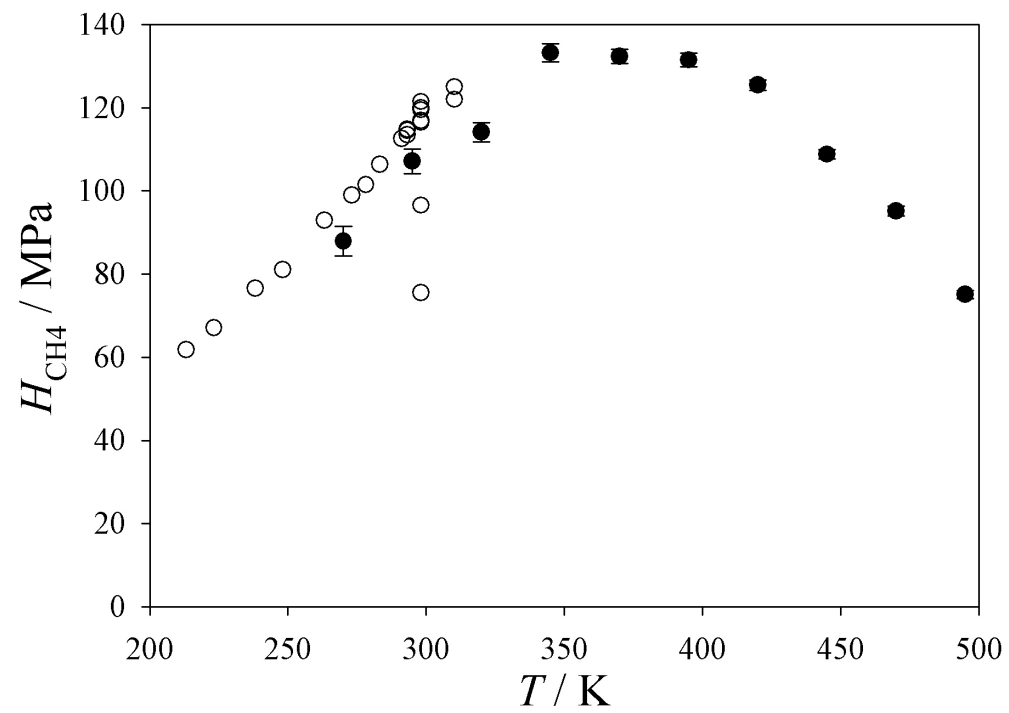

Fig. 2. Henry's law constant of methane in methanol: simulation data $(\bullet)$ and experimental data $[12,13,14,15,16,17,18,19,20,21](\circ)$.

largest investigation in this direction. It was facilitated by the extensive computing equipment at the High Performance Computing Center Stuttgart.

In the next step, all 33 ternary mixtures of these 78 components for which experimental VLE data is available were studied by molecular simulation. No adjustment to ternary data was carried out at all so that the calculations were strictly predictive. By comparing to experimental data, it was found that these predictions are very reliable as there was practically always an excellent match. As an example, Fig. 3 shows the ternary system consisting of methane, ethane, and carbon dioxide. Again, the computational effort was substantial, publications in the literature by other groups in this field typically cover one to two mixtures only.

\section{Vapor-liquid interface cluster criteria}

A suitable cluster criterion should achieve two goals: on the one hand, it needs to distinguish the bulk liquid and the bulk vapor successfully in every case - even when the vapor is supersaturated, such as in the vicinity of a droplet, or the liquid is undersaturated, such as in the vicinity of a bubble. On the other hand, the cluster criterion should also minimize noise fluctuations of the detected clusters to emphasize the signal.

The following criteria for carbon dioxide were compared for this purpose using a rigid two-center LJ plus point quadrupole (2CLJQ) model [22]: 


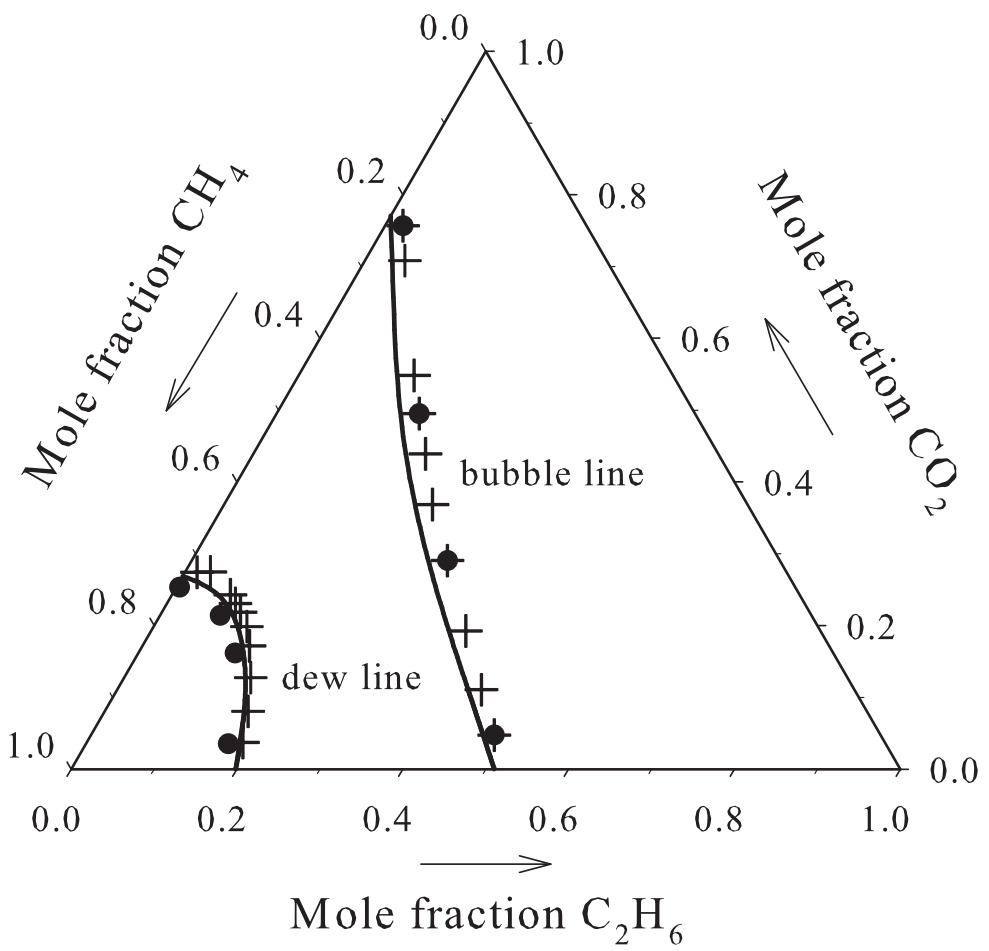

Fig. 3. Ternary vapor-liquid equilibrium phase diagram for the mixture $\mathrm{CH}_{4}+\mathrm{CO}_{2}+\mathrm{C}_{2} \mathrm{H}_{6}$ at $230 \mathrm{~K}$ and $4650 \mathrm{kPa}$ : simulation data $(\bullet)$, experimental data (+) of Knapp et al. [24], and Peng-Robinson equation of state (-).

- Stillinger [25]: all molecules with a distance of $r_{\mathrm{St}}$ or less from each other are liquid and belong to the same cluster (i.e., the same liquid phase or the same droplet). The Stillinger radius was set to $r_{\mathrm{St}}=5.7 \AA$ for the present simulations.

- Ten Wolde and Frenkel [26]: all molecules with at least $k$ neighbors within a distance of $r_{\mathrm{St}}$ belong to the liquid. They belong to the same cluster as all other liquid molecules within a distance of $r_{\mathrm{St}}$.

- Arithmetic mean, $k$ neighbors: a molecule is liquid if the density in the sphere containing its $k$ nearest neighbors exceeds $\left(\rho^{\prime}+\rho^{\prime \prime}\right) / 2$, where $\rho^{\prime}$ and $\rho^{\prime \prime}$ are the saturated liquid and vapor density, respectively. The molecule belongs to the same cluster as all other liquid molecules within the radius $r_{k}$, which defines a sphere with the volume occupied by $k+1$ molecules at a density of $\left(\rho^{\prime}+\rho^{\prime \prime}\right) / 2$.

- Geometric mean, $k$ neighbors: analogous, with a density threshold of $\left(\rho^{\prime} \rho^{\prime \prime}\right)^{1 / 2}$.

The simulations were conducted with the massively parallel MD program $l s 1$ mardyn (the precursor implementation ls 1 moldy is described by Bernreuther and Vrabec 
[27]). Figure 4 shows that all of the discussed criteria are applicable. The Stillinger criterion and the geometric mean density criterion with two neighbors lead to the best results. It should be noted that at high temperatures, i.e., near the critical point, the Stillinger criterion becomes less reliable in distinguishing the liquid from a supersaturated vapor than the geometric mean density criterion.

\section{Contact angle dependence on the fluid-wall interaction}

In cases where no hydrogen bonds are formed between the wall and the fluid, vaporsolid and liquid-solid interfacial properties mainly depend on the fluid-wall dispersive interaction, even for hydrogen bonding fluids. The truncated and shifted LJ (TSLJ) potential [28]

$$
u_{i j}^{\mathrm{TSLJ}}\left(r_{i j}\right)= \begin{cases}4 \varepsilon\left[\sigma^{12}\left(r_{i j}^{-12}-r_{\mathrm{c}}^{-12}\right)+\sigma^{6}\left(r_{\mathrm{c}}^{-6}-r_{i j}^{-6}\right)\right] & r_{i j}<r_{\mathrm{c}} \\ 0 & r_{i j} \geq r_{\mathrm{c}},\end{cases}
$$

with a cutoff radius of $r_{\mathrm{c}}=2.5 \sigma$ accurately reproduces the dispersive interaction if adequate values for the size and energy parameters $\sigma$ and $\varepsilon$ are specified. Due to the relatively small cutoff radius, all computations are accelerated, while the descriptive

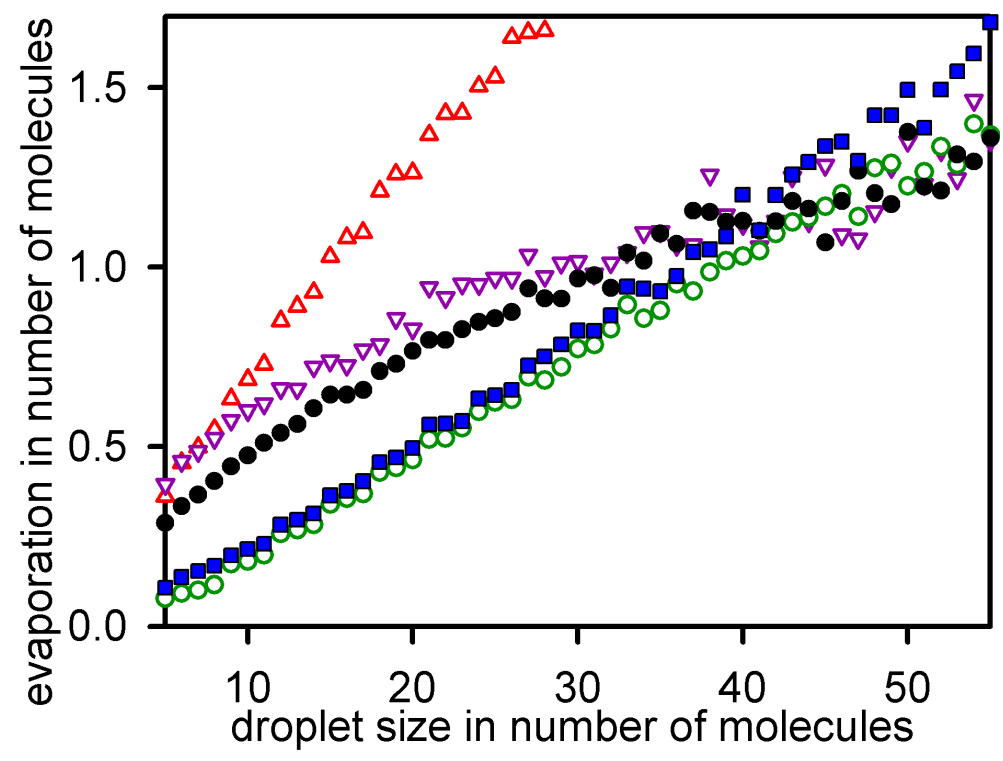

Fig. 4. Average number of molecules evaporating during a detection interval of $30 \mathrm{fs}$ from droplets in a supersaturated vapor of carbon dioxide with $T=237 \mathrm{~K}, \rho=1.98 \mathrm{~mol} / \mathrm{l}$, and $N$ $=1,050,000$, determined according to various cluster criteria: Stillinger $(\circ)$, ten Wolde and Frenkel with $k=4(\bullet)$, arithmetic mean with two $(\Delta)$ and eight neighbors $(\nabla)$ as well as geometric mean with two neighbors $(\square)$. 
power of the full LJ potential (without a cutoff) is retained even for systems with phase boundaries [29].

For the present series of contact angle simulations, the TSLJ model - with the potential parameters for methane, $\sigma=3.7241 \AA$ and $\varepsilon / k=175.06 \mathrm{~K}$ - was studied, extending a previous investigation of the vapor-liquid interface of the TSLJ fluid [29]. The TSLJ potential with the size and energy parameters $\sigma^{\prime}=\sigma$ as well as

$$
\varepsilon^{\prime}=\xi \varepsilon,
$$

was also applied for the unlike interaction between the fluid molecules and the wall atoms, with the same cutoff radius as for the fluid. The wall was modeled as a system of coupled harmonic oscillators with different spring constants for transverse and longitudinal motion, adjusted to simulation results for graphite that were obtained with a rescaled version of the Tersoff [30] potential.

The simulations were carried out with the $l s 1$ mardyn program. Vapor and liquid were independently equilibrated in homogeneous simulations for $10 \mathrm{ps}$. This was followed by $200 \mathrm{ps}$ of equilibration for the combined system, i.e., a liquid meniscus surrounded by vapor, with a graphite wall consisting of four to seven layers, cf. Fig. 5. A periodic boundary condition was applied to the system, leaving a channel with a diameter of $27 \sigma$ between the wall and its periodic image. The contact angle was determined from the density profiles by averaging over at least 800 ps after equilibration.
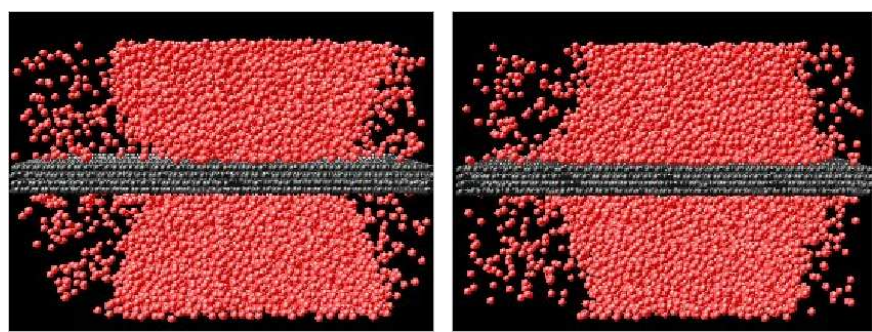

Fig. 5. Simulation snapshots for a reduced fluid-wall dispersive energy $\xi$ of 0.07 (left) and 0.13 (right) at a temperature of $0.88 \xi / k$.

Liquid menisci between graphite walls were simulated for a reduced fluid-wall dispersive energy $\xi$ between 0.07 and 0.16 at temperatures of $0.73,0.88$, and $1 \varepsilon / k$. Note that the triple point temperature of the TSLJ fluid is about $0.65 \varepsilon / k$ [31] while the critical temperature is $T_{\mathrm{c}}=1.0779 \varepsilon / k$ [29], so that the entire regime of stable vapor-liquid coexistence was covered.

High values of $\xi$ correspond to a strong attraction between fluid and wall compoents, leading to a contact angle $\vartheta$ smaller than $90^{\circ}$, i.e., to partial $\left(\vartheta>0^{\circ}\right)$ or full $\left(\vartheta=0^{\circ}\right)$ wetting of the surface. For a higher fluid-wall dispersive energy, the extent of wetting increases, cf. Fig. 5. The transition from obtuse to acute contact angles, i.e., $\vartheta\left(T, \xi_{0}\right)=90^{\circ}$, occurs at a temperature independent value $\xi_{0} \approx 0.113$ of the 
fluid-wall dispersive energy, as can be seen in Fig. 6. Furthermore, the symmetry law

$$
\cos \vartheta\left(T, \xi_{0}-\Delta \xi\right)=-\cos \vartheta\left(T, \xi_{0}+\Delta \xi\right),
$$

is valid over the whole relevant range of temperatures and magnitudes of the fluidwall dispersive energy. Figure 6 also shows that there is a narrow range of $\xi$ values that lead to the formation of a contact angle, as opposed to total dewetting or wetting. As the temperature decreases and the vapor-liquid surface tension $\gamma(T)$ increases, the contact angle approaches $90^{\circ}$.

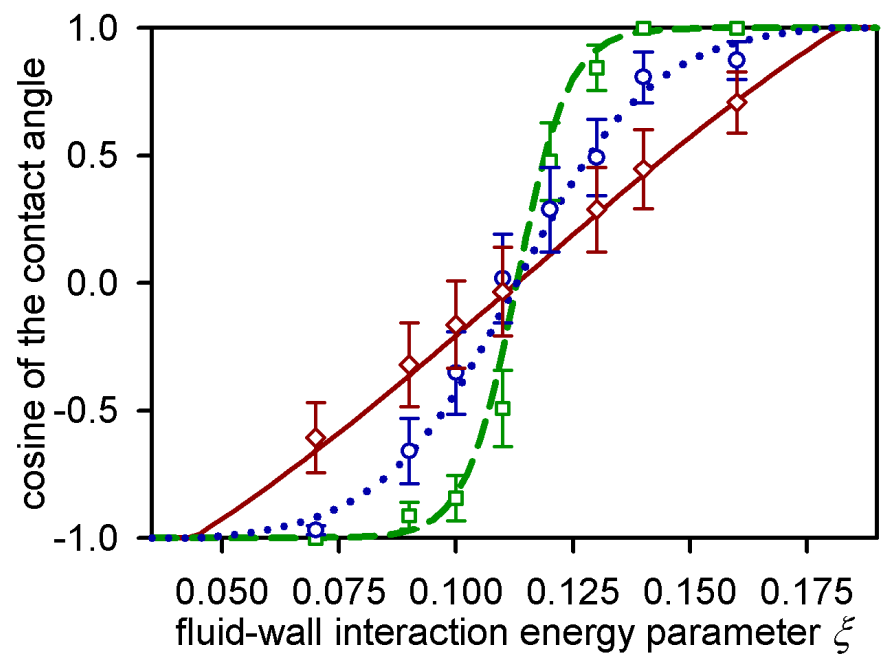

Fig. 6. Simulation results and correlation for the contact angle in dependence of the reduced fluid-wall dispersive energy $\xi$ at temperatures of 0.73 (diamonds and solid line), 0.88 (circles and dotted line) as well as 1 (squares and dashed line) $\varepsilon / k$.

\section{A sterically accurate generic benzyl alcohol model}

Benzyl alcohol $\left(\mathrm{C}_{6} \mathrm{H}_{5}-\mathrm{CH}_{2} \mathrm{OH}\right)$ is widely used as a solvent for paints and inks. However, it is classified as a harmful substance $(\mathrm{Xn})$ and should not be inhaled, nor used at high temperatures where it exhibits a high vapor pressure.

The basis for a new rigid molecular model of benzyl alcohol was determined by $a b$ initio calculations with the GAMESS (US) quantum chemistry package [32], obtaining the quadrupole moment as well as the equilibrium positions of the nuclei as illustrated in Fig. 7.

Further potential parameters were taken from accurate empirical molecular models for related fluids, leading to a sterically accurate generic model, cf. Tab. 1, that can be used as a starting point for parameter optimization. In particular, point charges 
as well as $\sigma$ and $\varepsilon$ parameters for the hydroxyl group were taken from the ethanol model of Schnabel et al. [33]. Moreover, the $\sigma$ and $\varepsilon$ values of the corresponding LJ interaction site of the Merker et al. [34] cyclohexanol model were used for the $\mathrm{CH}_{2}$ group, while the $\mathrm{LJ}$ parameters for the $\mathrm{CH}$ and $\mathrm{C}$ centers were set according to the Huang et al. [35] models of benzene and phosgene, respectively.

Table 1. Coordinates and parameters of the LJ sites, and the point charges, and the point quadrupole for the present benzyl alcohol model. Bold characters indicate represented atoms.

\begin{tabular}{lccccccc}
\hline \hline Interaction & $x$ & $y$ & $z$ & $\sigma$ & $\varepsilon / k_{\mathrm{B}}$ & $q$ & $Q$ \\
site & \multicolumn{1}{c}{$\AA$} & $\AA$ & $\AA$ & $\AA$ & $\mathrm{K}$ & $e$ & $D \AA$ \\
\hline $\mathbf{C H}^{\mathrm{p}}$ & 0.695 & -0.037 & 2.218 & 3.247 & 88.97 & & \\
$\mathbf{C H}^{1, \mathrm{~m}}$ & -0.753 & -0.204 & 1.941 & 3.247 & 88.97 & & \\
$\mathbf{C H}^{\mathrm{r}, \mathrm{m}}$ & 1.638 & 0.189 & 1.090 & 3.247 & 88.97 & & \\
$\mathbf{C H}^{1, \mathrm{o}}$ & -1.255 & -0.149 & 0.543 & 3.247 & 88.97 & & \\
$\mathbf{C H}^{\mathrm{r}, \mathrm{o}}$ & 1.134 & 0.250 & -0.304 & 3.247 & 88.97 & & \\
$\mathbf{C}$ & -0.285 & 0.080 & -0.497 & 2.810 & 10.64 & & \\
$\mathbf{C H}$ & -1.160 & 0.372 & -2.039 & 3.412 & 102.2 & +0.2556 & \\
$\mathbf{O H}$ & 0.070 & -0.516 & -2.835 & 3.150 & 85.05 & -0.6971 & \\
OH & 0.191 & -1.430 & -2.614 & & & +0.4415 & \\
Benzyl & 0 & 0 & 0 & & & & 2.534 \\
\hline \hline
\end{tabular}

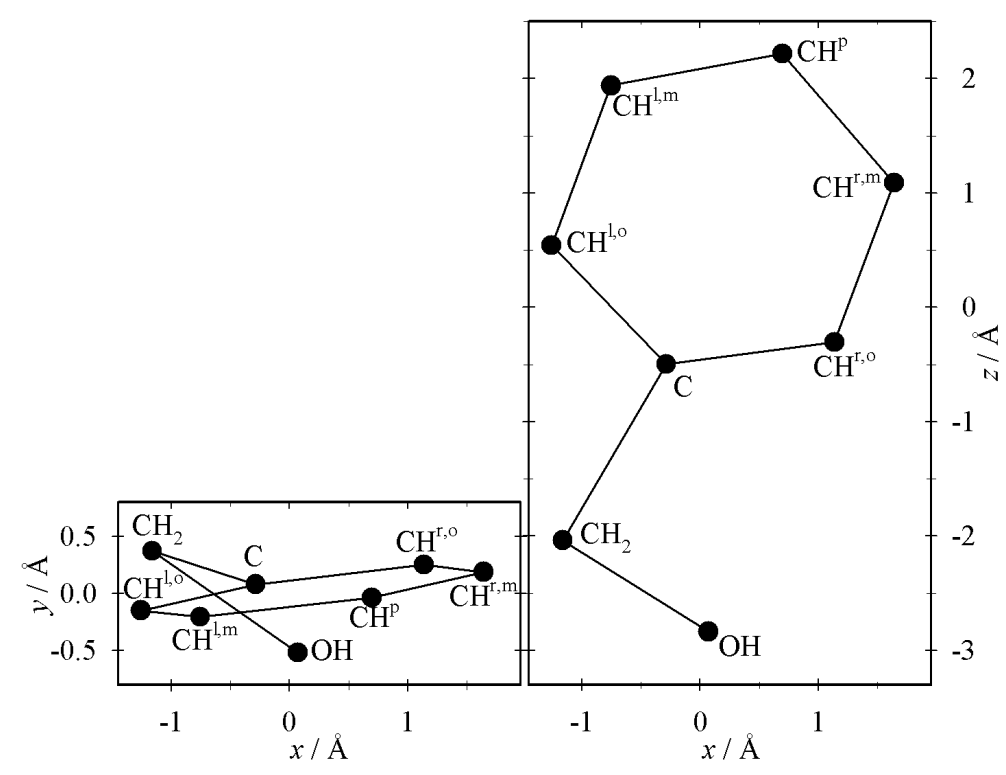

Fig. 7. Coordinates of the LJ sites for the present benzyl alcohol model. 


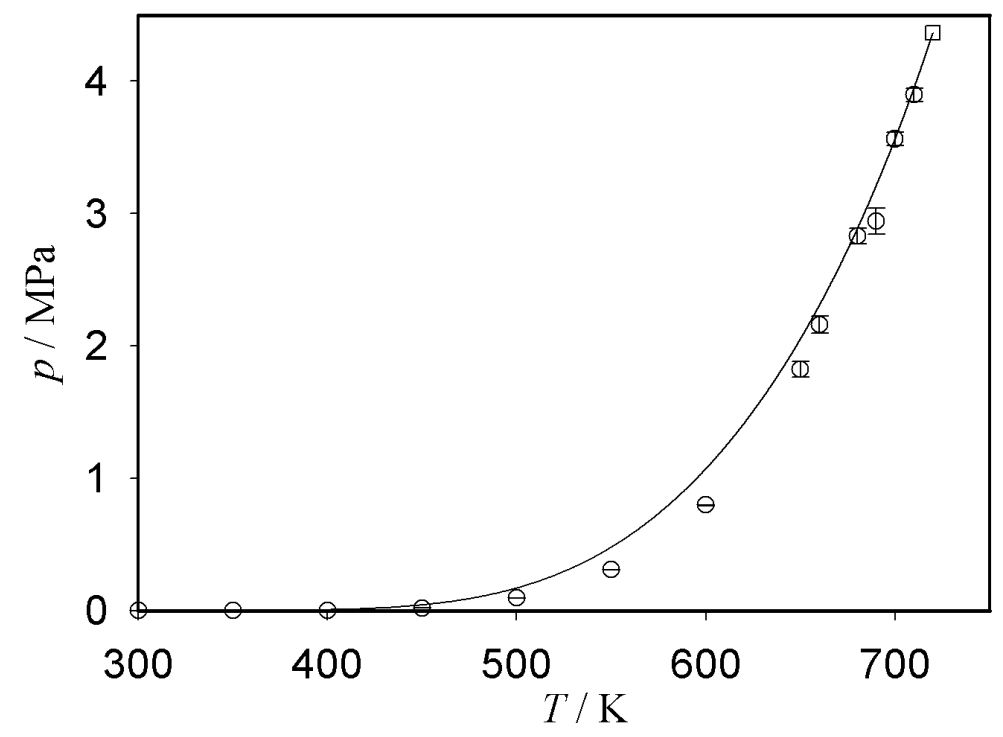

Fig. 8. Vapor pressure of benzyl alcohol according to a correlation [36] based on experiments $(-)$ as well as present molecular simulation data (०).

VLE properties of the generic model were calculated using the ms2 program, leading to an overall satisfactory first approximation, considering that all a posteriori adjustments were absent. The vapor pressure is more accurate at high temperatures, cf. Fig. 8.

\section{Computing performance}

The scalability of the $l s 1$ mardyn program was measured on the cacau supercomputer for simulation scenarios involving methane, represented by the TSLJ fluid, as well as graphite, modeled by a rescaled version of the Tersoff [30] potential. MPI parallelization was applied according to a spatial domain decomposition scheme with equally sized cuboid subdomains and a cartesian topology based on linked cells [27].

Often the best solution is an isotropic decomposition that minimizes the surface to volume ratio of the spatial subdomains. For the simulation of homogeneous systems, this approach is quite efficient. That is underlined by the weak and strong scaling behavior of ls 1 mardyn for typical configurations, shown in Fig. 9 (left), in cases where supercritical methane ('fluid') at a density of $10 \mathrm{~mol} / \mathrm{l}$ and solid graphite ('wall') were considered with a system size of up to 4,800,000 interaction sites, representing the same number of carbon atoms and methane molecules here. Graphite simulations, containing only carbon atoms, scale particularly well, due to a favorable relation of the delay produced by communication between processes to the concurrent parts, i.e., the actual intermolecular interaction computation, which is much more expensive for the Tersoff potential than the TSLJ potential. 

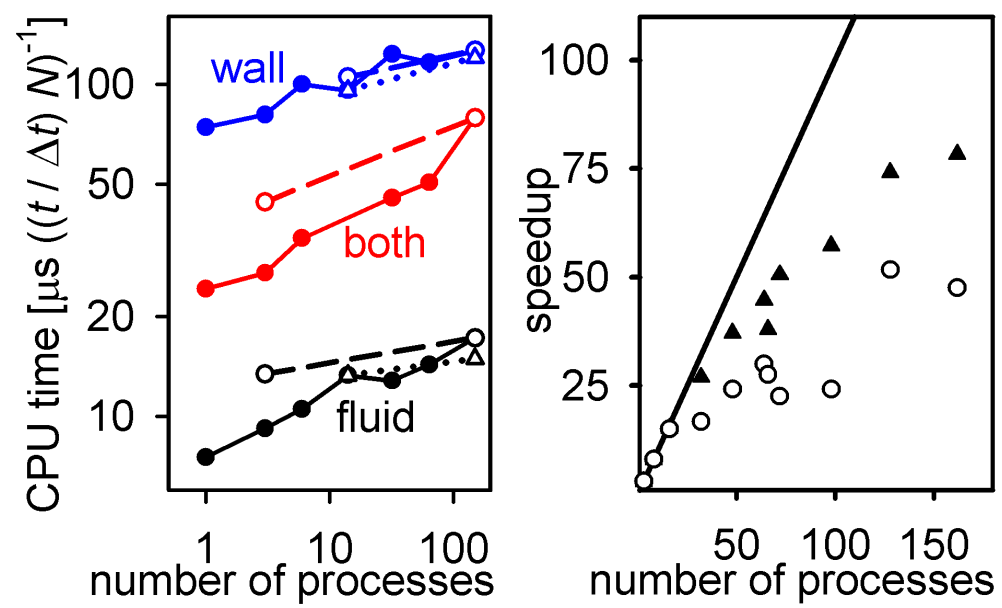

Fig. 9. Left: Total CPU time, i.e., execution time multiplied with the number of parallel processes, per time step and interaction site for weak scaling with 3,000 (dashed lines / circles) and 32,000 (dotted lines / triangles) interaction sites per process as well as strong scaling with 450,000 interaction sites (solid lines / bullets), using isotropic spatial domain decomposition. Right: speedup for a system of liquid methane between graphite walls with 650,000 interaction sites, where isotropic (circles) and channel geometry based (triangles) spatial domain decomposition was used; the solid line represents linear speedup.

The simulation of combined systems, containing both fluid and solid interaction sites, is better handled by a channel geometry based decomposition scheme, where an approximately equal portion of the wall and a part of the fluid is assigned to each process, cf. Fig. 9 (right). In the general case, where spatial non-uniformities do not match any cartesian grid, a flexible topology has to be used. An approach based on $k$-dimensional trees [37], implemented in a version of $l s 1$ mardyn, showed clearly improved results with respect to the scaling of inhomogeneous systems.

\section{Conclusion}

The intermolecular interaction of small hydrogen bonding molecules like mono- and dimethylamine can be described by simple LJ based united-atom molecular models with point charges. Such computationally efficient models were applied to binary mixtures with non-hydrogen bonding components regarding VLE properties. Accurate predictions, covering a broad range of temperatures and compositions, were obtained, regardless whether the state independent binary interaction parameter was adjusted to Henry's law constant or vapor pressure.

For a two-center LJ plus quadrupole model of carbon dioxide, a comparison of cluster criteria with the purpose of accurately detecting the vapor-liquid phase boundary gave overall support to the geometric mean density criterion applied to the sphere consisting of a molecule and its two nearest neighbours. The Stillinger criterion was 
found to be particularly adequate at low temperatures. For the TSLJ fluid, the contact angle formed between the vapor-liquid interface and a wall was determined by canonical ensemble MD simulation while the mangitude of the dispersive fluid-wall interaction was varied. Over the whole temperature range between triple point and critical point, the contact angle dependence on the fluid-wall dispersive energy obeys a simple symmetry law.

A sterically realistic model for benzyl alcohol was presented, showing within the framework of LJ sites with point polarities and electric point charges that the generic molecular modeling approach can lead to a good starting point for parameter optimization with respect to VLE properties such as the vapor pressure.

The scalability of the lsl mardyn program was assessed and found to be acceptable. MD simulations of methane confined between graphite walls with up to 4,800,000 interaction sites, i.e., carbon atoms and methane molecules, were conducted to demonstrate the viability of the program.

The authors would like to thank Martin Bernreuther, Martin Buchholz, Domenic Jenz, and Christoph Niethammer for contributing to the $l s 1$ mardyn code, Stephan Deublein, Bernhard Eckl, Gimmy Fernández Ramírez, Gabriela Guevara Carrión, and Sergei Lishchuk for contributing to the ms2 code, Ioannis Pitropakis for performing simulation runs of binary mixtures, as well as Calin Dan, Franz Gähler, Sebastian Grottel, Jens Harting, Martin Hecht, Ralf Kible, and Guido Reina for frank discussions and their persistent support.

\section{References}

1. Th. Schnabel, J. Vrabec, and H. Hasse. Unlike Lennard-Jones parameters for vapor-liquid equilibria. J. Mol. Liq., 130:170-178, 2007.

2. J. Vrabec, J. Stoll, and H. Hasse. Molecular models of unlike interactions in fluid mixtures. Mol. Sim., 31:215-221, 2005.

3. Ph. Ungerer, C. Nieto-Draghi, B. Rousseau, G. Ahunbay, and V. Lachet. Molecular simulation of the thermophysical properties of fluids: From understanding toward quantitative predictions. J. Mol. Liq., 134:71-89, 2007.

4. Th. Schnabel, J. Vrabec, and H. Hasse. Molecular simulation study of hydrogen bonding mixtures and new molecular models for mono- and dimethylamine. Fluid Phase Equilib., 263:144-159, 2008.

5. J.Vrabec, Y.-L. Huang, and H. Hasse. Molecular models for 267 binary mixtures validated by vapor-liquid equilibria: A systematic approach. Fluid Phase Equilib., 279(2):120-135, 2009.

6. T. Werder, J. H. Walter, R. L. Jaffe, T. Halicioglu, and P. Koumoutsakos. On the watercarbon interaction for use in molecular dynamics simulations of graphite and carbon nanotubes. J. Phys. Chem. B, 107:1345-1352, 2003.

7. G. F. Teletzke, L. E. Scriven, and H. T. Davis. Wetting transitions. II. First order or second order? J. Chem. Phys., 78:1431-1439, 1982.

8. J. Wedekind and D. Reguera. What is the best definition of a liquid cluster at the molecular scale? J. Chem. Phys., 127:154516, 2007.

9. Th. Schnabel, M. Cortada, J. Vrabec, S. Lago, and H. Hasse. Molecular model for formic acid adjusted to vapor-liquid equilibria. Chem. Phys. Lett., 435:268-272, 2007. 
10. J. H. Hong, P. V. Malone, M. D. Jett, and R. Kobayashi. The measurement and interpretation of the fluid-phase equilibria of a normal fluid in a hydrogen bonding solvent: the methane-methanol system. Fluid Phase Equilib., 38:83-96, 1987.

11. N. L. Yarym-Agaev, R. P. Sinyavskaya, I. I. Koliushko, and L. Y. Levinton. J. Appl. Chem. USSR, 58:154-157, 1985.

12. M. G. Levi. Gazzetta Chimica Italiana, 31:513-541, 1901.

13. A. S. McDaniel. The absorption of hydrocarbon gases by nonaqueous liquids. J. Phys. Chem., 15:587-610, 1911.

14. L. S. Bezdel and V. P. Teodorovich. Gazovaya Prom., 8:38-43, 1958.

15. F. L. Boyer and L. J. Bircher. The solubility of nitrogen, argon, methane, ethylene and ethane in normal primary alcohols. J. Phys. Chem., 64:1330-1331, 1960.

16. A. Lannung and J. C. Gjaldback. The solubility of methane in hydrocarbons, alcohols, water and other solvents. Acta Chem. Scand., 14:1124-1128, 1960.

17. E. R. Shenderei, Y. D. Zelvenskii, and F. P. Ivanovskii. Gazovaya Prom., 6:42-45, 1961.

18. J. Tokunaga and M. Kawai. Solubilities of methane in methanol-water and ethanol-water solutions. J. Chem. Eng. Jap., 8:326-327, 1975.

19. J. Makranczy, L. Rusz, and K. Balogmegyery. Solubility of gases in normal alcohols. Hung. J. Ind. Chem., 7:41-46, 1979.

20. P. Tkaczuk. Experimentelle Bestimmung der Gaslöslichkeit von Methan in Ketonen und Vergleich mit vorausberechneten Ergebnissen. Technical report, Technische Universität Dortmund, 1986.

21. S. Bo, R. Battino, and E. Wilhelm. Solubility of gases in liquids. 19. solubility of $\mathrm{He}, \mathrm{Ne}$, $\mathrm{Ar}, \mathrm{Kr}, \mathrm{Xe}, \mathrm{N}_{2}, \mathrm{O}_{2}, \mathrm{CH}_{4}, \mathrm{CF}_{4}$, and $\mathrm{SF}_{6}$ in normal 1-alkanols $\mathrm{n}^{-} \mathrm{C}_{\ell} \mathrm{H}_{2 \ell} \mathrm{OH}(1 \leq \ell \leq 11)$ at 298.15 K. J. Chem. Eng. Data, 38:611-616, 1993.

22. J. Vrabec, J. Stoll, and H. Hasse. A set of molecular models for symmetric quadrupolar fluids. J. Phys. Chem. B, 105:12126-12133, 2001.

23. J. Stoll, J. Vrabec, and H. Hasse. A set of molecular models for carbon monoxide and halogenated hydrocarbons. J. Chem. Phys., pages 11396-11407, 2003.

24. H. Knapp, X. Yang, and Z. Zhang. Vapor-liquid equilibria in ternary mixtures containing nitrogen, methane, ethane and carbondioxide at low temperatures and high pressures. Fluid Phase Equilib., 54:1-18, 1990.

25. F. H. Stillinger. Rigorous basis of the Frenkel-Band theory of association equilibrium. $J$. Chem. Phys., 38:1486-1494, 1963.

26. P. R. ten Wolde and D. Frenkel. Computer simulation study of gas-liquid nucleation in a Lennard-Jones system. J. Chem. Phys., 109:9901-9918, 1998.

27. M. Bernreuther and J. Vrabec. Molecular simulation of fluids with short range potentials. In M. Resch et al., editors, High Performance Computing on Vector Systems, pages 187195, Heidelberg, 2006. Springer.

28. M. P. Allen and D. J. Tildesley. Computer Simulation of Liquids. Clarendon, Oxford, 1987.

29. J. Vrabec, G. K. Kedia, G. Fuchs, and H. Hasse. Comprehensive study on vapour-liquid coexistence of the truncated and shifted Lennard-Jones fluid including planar and spherical interface properties. Mol. Phys., 104:1509-1527, 2006.

30. J. Tersoff. Empiric interatomic potential for carbon, with applications to amorphous carbon. Phys. Rev. Lett., 61:2879-2882, 1988.

31. J. A. van Meel, A. J. Page, R. P. Sear, and D. Frenkel. Two-step vapor-crystal nucleation close below triple point. J. Chem. Phys., 129:204505, 2008.

32. M. W. Schmidt, K. K. Baldridge, J. A. Boatz, S. T. Elbert, M. S. Gordon, J. H. Jensen, Sh. Koseki, N. Matsunaga, K. A. Nguyen, Sh. Su, Th. L. Windus, M. Dupuis, and J. A. 
Montgomery jr. General atomic and molecular electronic structure system. J. Comp. Chem., 14:1347-1363, 1993.

33. Th. Schnabel, J. Vrabec, and H. Hasse. Henry's law constants of methane, nitrogen, oxygen and carbon dioxide in ethanol from 273 to $498 \mathrm{~K}$ : Prediction from molecular simulation. Fluid Phase Equilib., 233:134-143, 2005. See also Fluid Phase Equilib. 236: 272 (2005) and 239: 125-126 (2006).

34. Th. Merker, G. Guevara-Carrión, J. Vrabec, and H. Hasse. Molecular modeling of hydrogen bonding fluids: New cyclohexanol model and transport properties of short monohydric alcohols. In Wolfgang E. Nagel et al., editors, High Performance Computing in Science and Engineering '08, Transactions of the High Performance Computing Center, Stuttgart, pages 529-541, Heidelberg, 2009. Springer.

35. Y.-L. Huang, H. Hasse, and J. Vrabec. Molecular modeling of industrially relevant aromatic fluids. 2009.

36. R. L. Rowley, W. V. Wilding, J. L. Oscarson, Y. Yang, N. A. Zundel, T. E. Daubert, and R. P. Danner. DIPPR R Data Compilation of Pure Compound Properties. AIChE, New York, 2006.

37. J. L. Bentley. Multidimensional binary search trees used for associative searching. CACM, 18(9):509-517, 1975. 